Terrains \& travaux, $\mathrm{n}^{\circ} 11$, décembre 2006, pp. 16-35

Robin Foot \& Ghislaine Doniol-Shaw

\title{
La dérive d'un dispositif de sécurité : de l'homme mort à l'homme incertain
}

Si les êtres humains parlent parfois de la technique, les objets techniques ont aussi leur mot à dire sur la manière d'être de ces humains. Mais parfois ces discours s'embrouillent dans des contradictions sans fin. On ne comprend plus ce qui est dit. On peut alors parler de dérive surtout s'il s'agit d'un dispositif de sécurité dont la vocation est de mettre en discipline les corps pour en surveiller les défaillances éventuelles et récupérer, le cas échéant, le fonctionnement du système. Que ce dispositif n'ait plus une théorie certaine de l'homme et la surveillance qu'il exerce perd fatalement de sa pertinence et de son efficacité. Tel est le cas actuel du dispositif d'«homme mort» dans le monde ferroviaire et, en particulier, dans les tramways. Cette appellation $d^{\prime}$ '« homme mort» en rappelle bien la vocation initiale et rend plus sensible sa dérive au fil du temps et des innovations. Caractériser cette dérive et en comprendre la mécanique, tel sont les enjeux de cet article.

\section{Le jeu du dispositif}

L'intérêt que l'on peut porter au concept de dispositif développé par Michel Foucault à partir de Surveiller et Punir (Foucault, 1975) ne se situe probablement pas tant dans la diffusion et l'extension de l'usage du terme luimême que par la nouvelle perspective qu'elle a ouverte pour l'analyse de nos sociétés. Pour l'essentiel, le dispositif foucaldien ne se préoccupe pas de réagencer un social et un technique, supposés dissociables, que de lier les mots 
aux choses. Il rompt ainsi avec la préoccupation des seules formations discursives, les épistémés (Foucault, 1966; Foucault, 1969), pour articuler, au sens propre comme au figuré (Latour, 1999), le discursif et le non discursif au sein d'un même réseau: "Un dispositif est un ensemble résolument hétérogène comportant des discours, des institutions, des aménagements architecturaux, des décisions réglementaires, des lois, des mesures administratives, des énoncés scientifiques, des propositions philosophiques, morales, philanthropiques, bref: du dit, aussi bien que du non dit, voilà les éléments du dispositif. Le dispositif lui-même, c'est le réseau qu'on peut établir entre ces éléments" (Foucault, 1994).

Que "l'énoncé soit indissociable de toutes les techniques, de tous les dispositifs, matériels et institutionnels, par lesquels les acteurs humains s'entredéfinissent" (Callon et Latour, 1982), sera un des apports essentiels de Michel Foucault et de son dispositif à la nouvelle sociologie des sciences. L'entrée des «non-humains » en société au travers de «l'acteur-réseau » en constituera, en quelque sorte, une suite logique. Mais dans cette suite, quelque chose s'est perdu, avec l'idée, portée par cette sociologie des sciences, que le sens de l'action peut être saisi à partir de l'interaction et de son résultat. Dans cette théorie, un acteur peut échouer s'il refuse de composer avec d'autres acteurs son projet (Latour, 1992) ou s'il ne parvient pas à stabiliser la coopération entre acteurs (Callon, 1986), mais s'il réussit dans son travail d'intéressement et d'association, alors le sens de l'action peut être rabattu sur le nouvel état du monde ainsi créé (Latour, 1989). Dans ce rabattement du sens de l'action sur l'entité produite, la «chose» qui s'est perdue, c'est l'existence d'un « jeu » possible, entre la prise en mots du dispositif et la prise au corps, entre les discours et les disciplines. Jeu qui conduit à ce que ce soit le dispositif lui-même qui puisse inventer alors sa propre fonction stratégique, fonction imprévue des acteurs dominants et restant souvent, pour eux, comme un point indicible.

Ainsi, la prison, considérée à un moment donné, comme un dispositif efficace de lutte contre la criminalité, s'avère avoir permis, in fine, "la constitution d'un milieu délinquant, très différent de cette espèce de semis de pratiques et d'individus illégalistes que l'on trouvait dans la société du XVIIIe siècle" (Foucault, 1994).

Ce jeu entre les différentes instances du réseau permet alors de comprendre pourquoi il peut y avoir un insu de l'action du dispositif sans qu'il soit nécessaire de recourir à "une ruse stratégique de quelque sujet méta-ou transhistorique qui l'aurait perç et voulu" (Foucault, 1994) ou à une raison cachée qu'il s'agirait 
de dévoiler. Cet insu est à chercher dans le réseau lui-même, à sa surface, dans les "savoirs assujettis », ces savoirs disqualifiés, ces savoirs locaux que l'on peut faire réapparaître et à partir desquels peut s'élaborer une critique (Foucault, 1997).

Si l'autonomie relative de l'action du dispositif vis-à-vis des intentions formulées par les humains et du sens qu'ils donnent à leur action, manifeste le jeu existant entre les éléments hétérogènes du réseau, elle traduit également que l'affirmation qu'un non-humain puisse être un acteur de premier plan ne correspond pas seulement à un effet de rhétorique mais est bien un fait de société.

Nous voudrions tenter de démontrer cette assertion à partir de l'analyse d'un dispositif de sécurité ferroviaire, le dispositif de veille communément appelé " homme mort», dont plus personne ne sait précisément comment sa forme traduit la fonction qui lui est assignée par décret, celle de provoquer "l'arrêt du train en cas de défaillance du mécanicien" (article 30 du décret du 22 mars 1942). Même au Ministère des transports, des experts scientifiques ont conclu, dans le cadre d'un appel d'offres sur la sécurité lancé par le Predit (Programme de recherche et d'innovation dans les transports terrestres), que: "Aucun système de veille n'a évité un accident" (e-mail du 7/12/2005 du secrétariat technique du GO4 du Predit).

Pourtant, les constructeurs continuent à les concevoir pour les implanter. Ils procèdent même à des innovations formelles et technologiques. Les services de l'État ont toujours en charge d'en contrôler la conformité et le bon fonctionnement. Des experts discutent minutieusement des paramétrages tandis que les conducteurs continuent à les faire fonctionner au risque d'avoir, parfois, des troubles musculo-squelettiques (TMS).

Dans cette dérive, le dispositif a entraîné à sa suite les acteurs humains. Ceuxci semblent impuissants à reprendre pied dans le réel de la fonction, à reprendre le dessus sur une forme technique. Une expertise «nouvelles technologies » demandée par le Comité d'entreprise du réseau de transport urbain de Clermont-Ferrand lors de l'introduction d'un tramway sur pneus a été l'occasion pour nous d'interroger ce dispositifl.

1 Un certain nombre de rapports d'enquête, de communications ou de publications sont disponibles à l'adresse suivante $: \underline{\text { http://latts.cnrs.fr/site/p lattsperso.php?Id=705 }}$ 


\section{L'effet d'estrangement d'un tramway sur pneu}

Certains objets semblent tellement faire partie d'un paysage qu'il ne vient à l'idée de personne de s'interroger sur leur place et leur forme. Leur présence est si forte qu'ils sont le paysage même. On ne les distingue plus véritablement. Pour percevoir de nouveau ces objets, il faut qu'un événement perturbe ce paysage, le dépayse à lui-même en quelque sorte. Alors, l'objet perd son caractère naturel, intangible, et peut être questionné à nouveaux frais. C'est ce procédé de "l'estrangement des choses", dont parle Chklovski, qui "semble susceptible de constituer un antidote efficace à un risque qui nous guette tous : celui de tenir la réalité (nous compris) pour sûre" (Ginzburg, 2001). À cette condition, l'évidence peut alors être interrogée.

Nous avons été confrontés à un exercice de ce type avec l'invention d'un nouveau véhicule pour les transports urbains, un tramway sur pneus produit par Lohr Industrie. Ce véhicule, transfuge récent du monde routier, en conserve quelques marques. Par plusieurs traits, son poste de conduite trahit cette origine hybride. Ainsi, comme dans un bus, le poste de conduite est à gauche et la commande de traction/freinage se fait à l'aide de deux pédales, alors que les tramways modernes ont un poste de conduite au centre de la cabine et une traction/freinage commandée par un manipulateur à l'instar de ce qui se fait pour les trains ou les métros.

L'appartenance de ce véhicule au monde des tramways, en dépit de ces marques routières résiduelles, implique sa soumission au référentiel normatif du ferroviaire. Il doit être, par conséquent, équipé comme les autres tramways, qui étaient soumis à l'article 30 du décret du 22 mars 1942, d'un «dispositif spécial provoquant l'arrêt du train en cas de défaillance du mécanicien ». C'est ce dispositif qui s'intitule, dans le langage ferroviaire, dispositif de veille, plus communément ou éloquemment appelé, «homme mort». Il existe une diversité de dispositifs de veille. Leur principe général est celui du maintien par le conducteur, au cours du trajet entre deux stations, d'un appui sur une commande manuelle ou au pied. Le relâchement de cet appui «signe » la défaillance du conducteur et entraîne le déclenchement du freinage d'urgence du véhicule. Dans certains cas, une temporisation brève (entre 2 et 3 secondes), associée à une alarme, permet au conducteur de rattraper un éventuel relâchement involontaire du dispositif de veille. Ce dispositif fait partie du paysage ferroviaire en France depuis l'électrification et les politiques 
de productivité amenant la suppression du deuxième agent susceptible de suppléer à une défaillance du conducteur. À la SNCF, ce mouvement date des années 50 et, à la RATP, des années 70 .

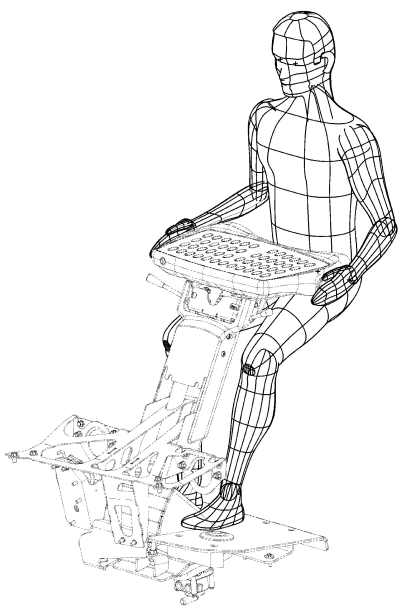

Modélisation de la position d'un conducteur à son poste de travail sur le Translohr (source : Lohr Industrie)

La présence de ce dispositif n'était pas prévue originellement car le véhicule était soumis au référentiel routier, c'est-à-dire au code de la route qui ne l'impose pas. Son ajout ne posait cependant pas, a priori, de problème particulier et, en l'absence de remontées négatives sur les systèmes existants, le constructeur du nouveau tramway sur pneus a choisi d'adopter l'architecture fonctionnelle des dispositifs à l'œuvre sur les plus récents tramways et d'assembler des éléments ayant déjà subi avec succès des procédures d'homologation. Il choisit ainsi d'implanter un dispositif de veille activé par une commande manuelle, sous la forme de boutons poussoirs situés de part et d'autre de la console de commande située devant le conducteur, à la place usuelle d'un volant. L'appui sur l'un ou l'autre de ces boutons réalise la fonction de veille.

Personne ne note que l'emprunt de ces différents éléments, validés et homologués sur d'autres types de matériels roulants, et l'implantation de cet "homme mort» dans ce nouveau tramway sur pneus, constituent une innovation dans la conception de ce dispositif et plus largement dans la conception du poste de conduite. Cette innovation, qui porte sur le travail de conduite, passe inaperçue aux yeux des concepteurs du véhicule essentiellement préoccupés par les performances du système technique et la conformité aux normes.

Pourtant, cette présence d'un «homme mort» activé manuellement sur un tramway à commande de traction/freinage par pédales constitue une «anomalie» dans le monde des tramways. En effet, s'il existe ou a existé toutes sortes d'associations entre la commande de traction/freinage et le dispositif de veille (veille associée au manipulateur de traction/freinage, avec

Robin Foot et Ghislaine Doniol-Shaw, "Dérive d'un dispositif » 
ou sans système d'activation au pied, action de veille et de traction/freinage par pédales), jamais, sur plus de cent ans d'histoire, on n'a conçu de tramway commandé par pédalier avec une veille actionnée à la main. Le plus étrange est que cette invention dont, a priori, on ne peut préjuger de la pertinence, se fasse quasiment par inadvertance, et par conséquent dans l'indifférence.

Tout se passe comme si, dès lors que le fonctionnement technique d'un dispositif d'«homme mort» est validé par les autorités compétentes et qu'il répond aux normes, il n'y a pas lieu de s'interroger sur les pratiques des conducteurs. Le mode de fonctionnement semble pouvoir se déduire essentiellement de sa forme, se réduire à une sorte de mode d'emploi écrit à partir des seuls plans de l'objet. Le fonctionnement, lié à la forme de l'objet technique, fait alors écran à la fonction qui ne se manifeste que dans l'usage effectif de l'objet par ses utilisateurs. La fonction quant à elle s'invente dans l’usage (Sigaut, 1991).

\section{Des objets et des hommes}

\section{Le silence des objets}

Cette indifférence à l'expérience accumulée dans l'histoire du monde ferroviaire ne concerne pas seulement le constructeur, mais touche l'ensemble des acteurs en charge de vérifier, contrôler et homologuer ce dispositif. Il n'y a en apparence rien à voir, rien à comprendre pourtant personne, ni le constructeur ni les services de l'État, n'est véritablement capable de justifier des options retenues tant pour la disposition des commandes que pour la logique fonctionnelle du dispositif. Il n'y a donc pas de théories précises ayant guidé l'invention de ce nouveau dispositif, juste des processus d'imitation, dans le respect des normes et des procédures d'homologation.

Cette absence de questionnement sur un dispositif de sécurité n'est pas extraordinaire. C'est au contraire banal. Dès lors qu'un dispositif ne fait pas parler de lui, c'est que tout va bien. "Qui ne dit mot consent» s'applique également aux choses.

Ce silence recouvre probablement plusieurs phénomènes différents voire opposés. 
Il y a d'abord le fait que la France redécouvre assez récemment le tramway (1985) et n'a donc plus d'expérience ni de compétence spécifique dans ce domaine. Un milieu professionnel est en train d'émerger et de se composer autour d'instances publiques nouvellement créées. Ce sont elles qui habilitent les experts et les organismes qualifiés. C'est donc depuis peu que s'organise la formalisation d'un retour d'expérience. Jusques là, l'expérience du tramway était essentiellement appropriée au sein de réseaux très personnalisés où l'expérience individuelle était prépondérante ${ }^{3}$.

Il y a ensuite le fait que les transports guidés sont relativement sûrs et qu'il y a assez peu d'accidents. Dans ce faible nombre d'événements, encore moins impliquent le dispositif de veille. De mémoire d'exploitant, pour ce qu'on a pu en connaître, personne ne se souvient d'une situation où le déclenchement de l'« homme mort» a servi à prévenir les conséquences d'une défaillance du corps. L'un d'entre eux précise même : "Moi qui suis à l'exploitation depuis 20 ans, je n'ai connaissance que d'incidents où il y a eu des malaises avec des conducteurs qui étaient arrêtés en station. Déjà, ils étaient arrêtés. Je ne peux pas dire qu'il n'y en a pas eu, mais en tout cas, moi, je n'en ai pas eu connaissance" 4 (Doniol-Shaw et Foot, 2006). Les circonstances du décès d'un conducteur du RER B, le 21 décembre 2004 sont venues confirmer ce constat. Celui-ci est mort peu après le départ de la Gare du Nord, mais il avait effectivement arrêté sa rame.

Enfin, il y a tous les incidents potentiellement liés à ce dispositif de sécurité qui ne font pas l'objet d'une formalisation permettant une prise en compte effective. Ainsi certains accidents restent connus seulement localement et ne font pas l'objet de déclaration. Dès lors qu'ils sont hors zone d'exploitation commerciale, ils peuvent passer inaperçus. Ce fut le cas, par exemple, pour un déraillement par dépassement du terminus à Grenoble. D’autres événements ne font l'objet d'aucune prise en compte comme les déclenchements

2 Ainsi le Service des remontées mécaniques et des transports guidés est créé en 2001 et la Commission nationale d'évaluation de la sécurité des transports guidés en 2003.

3 Sur ce point voir par exemple l'intervention d'Alain Séjourné, expert en tramway, dans les actes de la journée sur "Travail de conduite et sécurité des tramways : enjeux pour la conception du poste de conduite" (Doniol-Shaw et Foot, 2006) disponible à l'adresse suivante : http://latts.cnrs.fr/site/p_lattsperso.php?Id=767.

4 Didier Caligny, responsable, à la RATP, des études fonctionnelles sur le matériel roulant, (Doniol-Shaw et Foot, 2006). 
intempestifs de freinage d'urgence consécutifs à un «oubli» d'actionnement de l'«homme mort» par le conducteur. Pourtant, il serait intéressant de connaitre les causes de ces oublis car le déclenchement du freinage d'urgence n'est pas sans incidence sur la sécurité, la plupart des accidents de personnes ayant lieu à l'intérieur des rames. Il est probable que l'on s'apercevrait alors que le conducteur était pris par une autre action, plus urgente de son point de vue. D'autres encore sont bien reconnus comme étant liés au dispositif de veille mais ils ne rentrent pas dans la catégorie des événements mettant en cause la sécurité même s'ils affectent la santé des agents. Ce sont en particulier les troubles musculo-squelettiques, reconnus par la médecine du travail, occasionnés par la répétition à intervalles rapprochées d'un même geste pour activer la veille.

Pour analyser ce dispositif, il faut alors se départir d'une fascination pour l'exceptionnel, l'accident, et s'intéresser aux comportements usuels, afin de comprendre comment se constituent, dans l'ordinaire des situations de travail, l'appréhension de ces dispositifs (Amalberti et Barriquault, 1999). Au travers des formes concrètes d'appropriation de ces objets techniques, on voit se dessiner les écarts et inadéquations entre les normes et les pratiques. L'expression des conducteurs sur ces écarts permet alors de réexaminer ces objets.

\section{Les commandes de traction/freinage et l'homme mort, entre santé et sécurité}

À partir d'enquêtes dans quatre réseaux de transport (Marseille, Paris, SaintÉtienne et Strasbourg), d'une journée d'étude sur la sécurité rassemblant des acteurs de plusieurs réseaux et d'un séminaire sur quatre jours avec des syndicalistes, nous avons pu mettre à plat la diversité des systèmes d'homme mort sur la quasi totalité des réseaux de tramways existants (10 sur 11).

Un premier constat s'impose : dans tous les réseaux, le système d'homme mort est, en premier lieu, lié au système de commande de la traction et du freinage. Quand, il est manuel, c'est-à-dire sous la forme d'un manipulateur de traction, l'activation du système d'homme mort lui est associée directement. Quand les commandes sont au pied, par l'intermédiaire de deux pédales, l'une

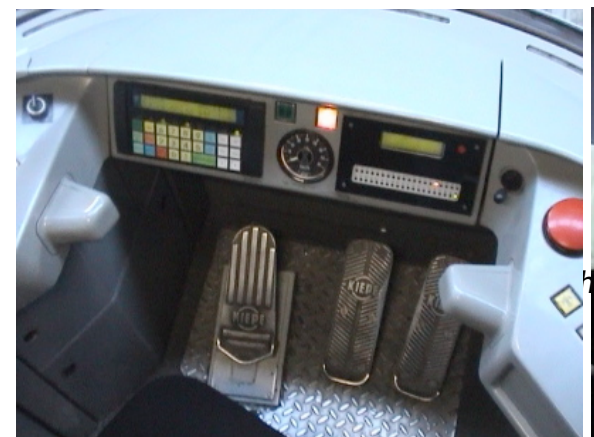

Poste de conduite du tramway de SaintÉtienne. À droite les pédales d'accélération et de freinage; à gauche, pédale d'activation de la veille

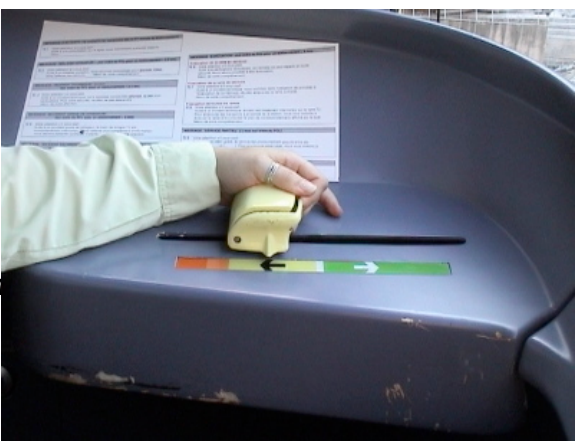

Manipulateur traction/freinage avec la commande de veille sur le Tramway Français Standard du réseau de la RATP. 
d'accélération, l'autre de freinage, actionnées toutes deux par le pied droit, l'activation de l'homme mort se fait également à l'aide d'une pédale, actionnée par le pied gauche.

Un second constat s'impose également: sur tous les réseaux qui sont dans une phase de renouvellement de matériel et dont les tramways sont équipés d'un manipulateur traction, il y a des demandes de transformation de ce manipulateur et de son dispositif d'« homme mort». Ces demandes formulées par les conducteurs ont été avalisées par la hiérarchie, signe tangible d'un accord sur le mauvais fonctionnement des systèmes existants. Elles ont conduit à des remaniements profonds du système, tant au niveau formel que technologique. L'exemple le plus spectaculaire a été celui de Nantes, où le manipulateur d'Alstom ${ }^{5}$ a été, dès le début, contesté par les conducteurs : "Les premiers conducteurs, sur la rame d'essai, avant même la mise en service, se sont plaints de ce système et on a rajouté une pédale, selon le système classique de la veille automatique où il faut maintenir la pédale à mi-course. Les conducteurs pouvaient choisir entre les deux systèmes de vigilance" 6

On observe que les modifications proposées vont toutes dans le sens d'une diminution des contraintes posturales induites par la forme du manipulateur traction qui mobilise en permanence une seule main dans plusieurs actions.

Certaines évolutions, comme à Strasbourg, ne jouent que sur la forme, passant d'un manipulateur rotatif à un joystick, sans qu'il soit envisagé de déconcentrer les commandes associées au manipulateur traction : débrayage du freinage d'urgence, sonnette et « homme mort».

D'autres, comme à Paris, modifient la forme, passant d'un manipulateur linéaire à un manipulateur rotatif équipé d'une commande sensitive pour la veille, tout en diversifiant les formes d'actionnement de l'« homme mort» par l'adjonction d'une pédale.

Dans ces deux cas, il n'y a pas d'analyse véritable sur les présupposés et les conséquences en matière de santé et de sécurité des choix faits. À Strasbourg,

\footnotetext{
5 Il est à noter que le manipulateur adopté pour le « tramway français standard » d'Alstom a été emprunté au métro lyonnais sans considération avec le fait que le pilotage automatique du métro rendait quasiment sans usage le manipulateur et la veille. Malgré les nombreux retours d'expérience critique sur l'ensemble des réseaux, on le retrouve sur les nouveaux tramwayx de Montpellier et de Bordeaux, vingt ans plus tard.

6 Alain Séjourné op.cit.
} 
il est à craindre que la reconduction d'une polarisation des commandes ne multiplie les contraintes sur le seul bras gauche, et ne réactive de ce fait les critiques formulées à l'encontre de l'ancien manipulateur. À Paris, si cette «libération» de la main de son obligation d'être sur le manipulateur traction diminue la contrainte, le fait que cela puisse induire un allongement du temps de réaction au freinage du conducteur n’a pas été examiné.

À l'opposé de ces expériences, le renouvellement du matériel de SaintÉtienne se caractérise par une grande stabilité dans la conception des commandes de traction/freinage et d'activation de la veille. Cette stabilité est d'autant plus remarquable que, à l'opposé des réseaux de Strasbourg ou de Paris, celui de Saint-Étienne a toujours exploité un tramway depuis la fin du XIX $^{\mathrm{e}}$ siècle (1881) et, depuis 1959, des tramways à commande par pédales.

Lors des discussions organisées avec les conducteurs pour définir le nouveau matériel, dans les années 1990, il n'y a pas eu de remontées négatives par rapport au système existant qui auraient fait pencher la balance en faveur d'un manipulateur traction. Le maintien des mêmes stéréotypes, sur les deux types de matériels qui devaient coexister sur la ligne de tramway, a alors prévalu pour reconduire cette option de commande par pédales. De la même manière, le système d' " homme mort » actionné par pédale a été reconduit.

Cette grande stabilité technique est un fait assez rare pour être souligné, d'autant plus que l'allure générale du poste de conduite de ce tramway à pédalier «moderne » ne ressemble pas à la génération précédente. Entre les deux, les changements de conception du poste de conduite apparaissent assez radicaux. On pourrait dire que tout a changé, y compris le design des pédales d'accélération et surtout de freinage, sauf la conception du système de veille.

Cette stabilité et la tendance à diversifier les possibilités d'actionnement de la veille par l'ajout d'une commande par pédale, quand la commande de traction se fait à l'aide d'un manipulateur, sont probablement autant d'indications que la mobilisation de la main pour actionner l'" homme mort » reste toujours problématique.

Les hypothèses de l' " homme mort » 
Comme tout objet technique, les systèmes de veille proposent un "script » d'action qui traduit les hypothèses de leurs concepteurs sur "les éléments qui composent le monde dans lequel l'objet est destiné à s'insérer" (Akrich, 1987). Le risque de la défaillance de l'homme est au cœur de ces dispositifs de sécurité, mais en examinant les différents systèmes nous sommes confrontés à une pluralité d'hypothèses, parfois contradictoires, sur les caractéristiques de la défaillance humaine. Pour comprendre ce paradoxe, il faut, au préalable, décrire ces différents systèmes et parvenir à expliciter les hypothèses de l'homme et de sa défaillance qu'elles inscrivent dans leur matérialité.

Deux stratégies de veille sont à l'oeuvre.

Dans la première, la veille automatique, le conducteur doit tout le temps activer la veille. Un relâchement se traduit, avec (Nantes) ou sans (SaintÉtienne) temporisation, par le déclenchement d'un freinage d'urgence.

Dans la seconde, le conducteur doit alternativement appuyer et relâcher la commande de veille, c'est la veille automatique à contrôle de maintien d'appui (Vacma) inventée dans les années 60. Les temporisations attribuées à l'une ou l'autre action varient suivant les réseaux, mais, dans la plupart (Paris, Grenoble, Lyon, Montpellier, Bordeaux...), les valeurs sont de l'ordre de douze secondes au maximum pour l'appui et de deux seconde au maximum, pour le relâché. Si un conducteur maintient l'appui plus de douze secondes sur la commande de veille ou s'il relâche cet appui plus de deux secondes, un buzzer retentit et, si aucune action sur la veille n'intervient dans les deux secondes, le freinage d'urgence se déclenche.

Un seul réseau, celui de Strasbourg, a conçu un dispositif qui considère indifféremment le maintien de l'appui ou le relâché. Le conducteur doit maintenir un même état au maximum huit secondes, sinon un premier avertissement sonore se déclenche puis, au bout de deux secondes sans modification d'état, une nouvelle sonnerie, avec un buzzer plus rapide que le premier, retentit et le conducteur a alors encore deux secondes pour changer l'état du dispositif, avant le déclenchement d'un freinage d'urgence.

Si ces dispositifs tentent de capter un même type d'information, un état de défaillance de l'homme, leur stratégie varie. Cette variation renvoie, de fait, à des théories différentes de l'homme que l'on peut expliciter ainsi. 
Avec la veille automatique, correspondant à un appui constant sur la commande, la théorie est simple. Si le conducteur a un malaise ou un endormissement profond, il se relâche et tout relâchement doit être interprété comme une défaillance. Le déclenchement du freinage d'urgence est alors le moyen de mettre le système en sécurité. Plus vite, ce relâchement est saisi, plus vite la mise en sécurité du véhicule peut être assurée.

La temporisation de deux secondes adoptée à Nantes entre le relâchement de la veille et le déclenchement du freinage d'urgence introduit une hypothèse seconde, celle d'un possible mauvais actionnement de la veille sans lien avec une défaillance physiologique du conducteur. Cette temporisation lui permet alors de récupérer une "fausse manœuvre ». La théorie de l'homme se complexifie d'une possibilité d'avoir seulement une défaillance de l'action et non du sujet. Mais le relâchement étant le signal convenu pour la défaillance, l'automate n'accorde que peu de temps à l'homme pour récupérer sa «mauvaise action ».

Avec la veille automatique avec contrôle du maintien d'appui, la Vacma, la théorie du dispositif se complique car, à la différence de la temporisation nantaise, elle n'autorise pas la défaillance de l'action mais, au contraire, l'oblige à intervalles non seulement réguliers mais en plus très rapprochés. La dissymétrie de la temporisation (12/2 secondes) formalise le fait que le relâchement plus que le maintien d'appui est considéré comme le signe potentiel d'une défaillance. Néanmoins, la fréquence de l'obligation de relâchement reste intrigante. Qu'est-ce qui justifie ces valeurs ?

Quand on interroge les experts du milieu ferroviaire, ils n'en savent rien. Plusieurs " théories indigènes " coexistent et circulent sur la rationalité de ce dispositif et de sa temporisation. Pour certains, le relâchement permettrait de s'assurer qu'un conducteur ne reste pas «agrippé » sur le manipulateur de traction alors qu'il aurait perdu connaissance. Pour d'autres, principalement du côté des constructeurs, cela permet d'éviter la « fraude ». Les différences de temporisation observées entre les trains $(55 / 5$ secondes), les métros (30/2 secondes) et les tramways (12/2 secondes) seraient liées aux différences de masse des rames, aux distances de freinage et aux intervalles de sécurité. Mais dès que l'on examine quelques instants cet argument, il s'avère sans consistance pratique car, dans un milieu urbain, quatre secondes suffisent largement pour brûler un feu rouge et traverser un carrefour. La théorie la plus souvent mobilisée pour justifier de 
la Vacma modifie de manière sensible sa fonction. Elle n'aurait pas tant pour but de prévenir une défaillance que de s'assurer de la vigilance du conducteur. Ce glissement de sens de veille à vigilance est assez fréquent et peut se retrouver, par exemple, dans des règlements de sécurité d'exploitation aussi bien que dans les discours d'experts?

Enfin, dernière hypothèse, sous-tendue par le fonctionnement de la Vacma de Strasbourg qui considère de manière symétrique l'appui et le relâchement, la défaillance de l'homme peut se manifester aussi bien par une crispation que par un relâchement.

\section{L'indicible hypothèse de la fraude?}

Nous avons donc vu quatre configurations du dispositif d'« homme mort ». Si trois d'entre elles ont une théorie facilement explicitable de leur fonctionnement, l'une nous laisse perplexe. La Vacma temporisée de manière dissymétrique en douze secondes pour l'appui et deux secondes pour le relâché n'a pas véritablement de théorie ou plutôt en a trop pour parvenir à être convaincante.

La théorie du contrôle de la vigilance qui est probablement celle qui est la plus souvent mobilisée pour justifier de ce système de Vacma, ne parvient pas à se fonder dans les faits. Régulièrement, on redécouvre que sa fonction n'est pas de contrôler la vigilance. Un accident récent est venu encore une fois le rappeler.

Le 30 août 2004 à Rouen une rame en percute une autre, arrêtée en station. L'enquête menée par le Bureau d'enquête accident des transports terrestres conclut ainsi : "La cause humaine est à l'origine de l'accident. L'hypothèse d'un malaise brutal a été étudiée dans un premier temps, et a laissé place à

7 Cf., par exemple, l'intervention de Laurent Vidal, expert de la Semaly, entreprise d'ingénierie ferroviaire, à la journée du 19 octobre 2004 (Doniol-Shaw et Foot, 2006). On peut aussi retrouver cette même confusion à l'étranger, comme dans ce livre de référence du monde ferroviaire britannique qui définit ainsi l'homme mort : "Un système plus sophistiqué fut conçu dans les années 60, habituellement défini comme dispositif de sécurité pour le conducteur ou de contrôle de vigilance. Son fonctionnement suppose que le conducteur manifeste sa vigilance en actionnant périodiquement un bouton du pupitre de commande ou en appuyant sur une pédale spécifique" in Simmons Jack \& Biddle Gordon (eds), 1997, The Oxford companion to British railway history, from 1603 to the 1990s, Oxford University Press, p.125. 
l'hypothèse beaucoup plus probable d'une hypovigilance du conducteur consécutive à un début de somnolence" ${ }^{8}$. La survenue de cet accident où un conducteur actionne au moins deux fois la Vacma, sans " voir» la rame devant lui, rappelle que "la seule information fiable qu'elle [la Vacma] peut délivrer sur le conducteur est la présence effective de ce conducteur sur la rame”.

Mais, si rien ne change, il est possible que cette conclusion soit encore une fois inaudible par le milieu en charge de la sécurité ferroviaire. En effet, cela fait près de quinze ans que l'on sait expérimentalement que les situations d'hypovigilance ne sont pas contradictoires avec le maintien d'une activité machinale. Ces travaux ont permis de constater " pendant ces phases de vigilance atténuée, un accroissement de la régularité dans les relâchés de la pédale et une augmentation de la durée du relâchement de l'appui. » Les chercheurs notent aussi que "c'est au cours de ces phases d'hypovigilance, qui doivent être considérées comme physiologiques, que nous avons observé les omissions de réponses aux signaux de limitation de vitesse ou aux signaux d'arrêt. " (Mollard, Coblentz et Cabon, 1991). Le système de veille peut ainsi être activé "normalement " par les conducteurs, sans que cela ne signifie pour autant qu'ils sont en situation de vigilance réelle.

Ce résultat est d'ailleurs quasi contenu dans le cahier des charges initial de la Vacma puisqu'une des trois conditions essentielles qu'elle devait satisfaire était "une mise en ouvre inconsciente du point de vue du conducteur qui devait être affranchi de la sujétion gênante que représentait une opération sans cesse réitérée" (Ribeill, 1997).

Pour comprendre cette capacité d'une « théorie » à rester dominante tout en ne résistant ni à l'épreuve des faits ni à celles des expériences scientifiques, il faut probablement en chercher l'explication du côté de ce cahier des charges élaboré par la SNCF dans les années 60. Dans ce document, il n'y a pas d'ambiguité. La Vacma, inventée en 1965, n'a pas pour fondement une controverse physiologique, mais un souci plus pragmatique des

8 Rapport d'enquête technique sur l'accident de tramway survenu à Rouen le 30 août 2004, Juin 2005 - rapport BEA-TT- $n^{\circ} 2004-007$. Ce document est disponible sur le site du Ministère : http://www.equipement.gouv.fr/article.php3?id_article=569 
ingénieurs de la SNCF d'éviter les " fraudes » qui avaient cours avec le dispositif de veille en usage sur les trains. Celui-ci consistait en un « cerclo », disposé sous le volant de traction, que le conducteur devait tenir collé au volant. Un simple lien pouvait être substitué à l'action manuelle, permettant ainsi au conducteur de se déplacer dans la cabine ce qui, pour des trajets de plusieurs heures sans arrêt, n'était pas négligeable. Le relâchement ne sert donc qu'à vérifier qu'il n'y a pas fraude sur l'information de maintien (Ribeill, 1997). La brièveté du temps de relâchement doit être interprétée comme un compromis nécessaire car le signal qui vérifie la conformité de l'action est le même que celui qui informe d'une perte de conscience.

L'émergence d'une théorie du contrôle de la vigilance, même si nous ne pouvons la dater avec précision, est donc postérieure à l'invention de la Vacma qui avait à la fois une hypothèse physiologique (la défaillance se traduit par le relâchement) et une hypothèse morale (l'homme fraude). Le fait qu'elle se soit substitué pour partie à la théorie originelle s'explique probablement par la difficulté pour les gestionnaires des réseaux de transport de légitimer durablement, aux yeux des agents, les contraintes imposées à leur travail par le souci de prévenir une fraude éventuelle.

Si on rétablit ce double fondement théorique du dispositif de l' '" homme mort » dans les formes prises par les défaillances physiologique et morale, on peut alors clarifier la controverse silencieuse, car enfouie dans la matérialité des dispositifs, qui traverse les réseaux de transports. Un simple tableau permet d'expliciter les hypothèses contradictoires sur l'homme et son comportement exprimées par les réseaux de transport urbain dans trois types de dispositifs.

Pour Nantes, Marseille et Saint-Étienne, la fraude n'est pas un problème et la défaillance de l'homme se traduit par un relâchement du corps. Un dispositif de veille par maintien constant d'appui est suffisant.

Pour Strasbourg, la fraude n'est pas un problème mais le comportement du corps humain est incertain. La défaillance peut se traduire par une crispation aussi bien que par un relâchement. Une Vacma avec une temporisation symétrique entre le maintien et le relâchement de l'appui est nécessaire. 
Pour Paris, Grenoble, Lyon, Bordeaux... la fraude est un problème et la défaillance se traduit par un relâchement du corps. Une Vacma avec une temporisation dissymétrique (temps de relâchement bref par rapport au temps d'appui) est nécessaire.

Une fois formulée sous cette forme, la controverse des dispositifs d' " homme mort » semblerait pouvoir être réglée assez facilement. Au regard de l'enjeu de sécurité publique, il suffirait alors de discuter de chacune des hypothèses.

La défaillance de l'homme se manifeste-t-elle par un relâchement ou une crispation ? En dehors de récits d'épouvante et de films d'horreur, l'hypothèse du relâchement est la seule que l'on puisse retenir d'un point de vue médical.

La fraude est-elle un problème ? Que ce soit à Marseille, à Saint-Étienne ou à Nantes personne n'a fait état d'un quelconque problème de fraude sur le dispositif de veille. Les différences entre les trains interurbains et le tramway peuvent expliquer cette situation. En premier lieu, les possibilités techniques de frauder à l'insu de la hiérarchie sont plus faibles car, les agents de maîtrise peuvent entrer dans le poste de conduite tout au long du trajet. En second lieu, les temps de trajet entre deux arrêts sont courts, de l'ordre de quelques minutes, et les conducteurs n'ont pas éprouvé la nécessité de frauder un dispositif ne leur posant pas problème.

Du point de vue de la sécurité, les dispositifs de veille automatique du type de celui de Saint-Étienne sont donc ceux qui garantissent la meilleure réactivité en cas de défaillance puisque la question de la fraude ne se pose pas dans le cadre des tramways urbains. Pourtant, ce sont les moins efficaces du point de la sécurité qui deviennent la norme.

\section{Conclusion : Le jeu du dispositif et l'indicible}

La dérive du dispositif de l'" homme mort» se manifeste dans ce mouvement qui tend à développer les systèmes les moins performants. Elle 
se manifeste également, sous d'autres formes, dans le monde ferroviaire. Ainsi, quand il faut définir les paramètres de ce dispositif pour des véhicules hybrides, circulant à la fois sur des lignes urbaines et des emprises ferroviaires, les tram-train ${ }^{9}$, personne ne sait comment le faire. En l'absence d'une explicitation et d'une confrontation des théories en jeu, la discussion tourne court et aboutit à un compromis comptable. La pertinence de l'hypothèse de la fraude n'est pas discutée. Le principe d'une Vacma est d'emblée retenue et, en l'absence de critère pour trancher les discussions, la négociation s'oriente vers un compromis, du type " couper la poire en deux », avec une temporisation " intermédiaire » entre le monde ferroviaire (55 secondes) et le monde des tramways (12 secondes). La temporisation de cette Vacma sera de 30 secondes pour le maintien et 2,5 secondes pour le relâchement.

Cette sorte de déréalisation du fonctionnement d'un dispositif de sécurité n'affecte pas seulement le monde des transports urbains de surface. Elle est encore plus fortement exprimée dans l'espace du métro ou du RER. Malgré les systèmes de contrôle continu de vitesse (de type Sacem sur le RER ou Ouragan sur le métro) et les contrôles de franchissement de signaux fermés qui surveillent en permanence que le mouvement d'un train est compatible avec ce qu'il peut rencontrer en aval, qui remplissent donc l'obligation édictée par l'article 30 du décret du 22 mars 1942, les dispositifs de veille, sous forme de Vacma, sont toujours là : "Avec un tel dispositif, on pourrait se demander à quoi sert un dispositif de veille ?"10. La seule réponse donnée est que les conducteurs ont toujours à les activer comme si, dans cette dérive du dispositif, celui-ci avait substitué à l'objectif de sécurité celui de la mise en discipline des conducteurs ${ }^{11}$.

La confirmation de cette tendance à la substitution de l'enjeu de sécurité par celui de la mise en discipline nous est donné par la procédure mise en place lors des défaillances des systèmes de contrôle du mouvement des

\footnotetext{
9 Des systèmes hybrides de tram-train vont être implantés à Mulhouse, Bondy-Aulnay et Montpellier.

10 On se reportera pour plus de précision sur cette situation de trouble cognitif à l'intervention de Didier Caligny, op.cit.

11 C'est seulement avec le pilotage automatique comme il existe sur les métros parisien, lyonnais et marseillais, que le conducteur n'a pas à actionner l' " homme mort ».
} 
trains. Les responsables du ferroviaire ont inventé pour cette occasion de nouvelles temporisations pour l'actionnement de la veille comme si l'innovation technologique avait induit également une transformation des formes de la défaillance des êtres humains. En cas de défaillances de ces systèmes de contrôle, au lieu de revenir aux temporisations antérieures, comme on pourrait s'y attendre, les responsables sont perplexes : "On s'est également demandé ce qui pourrait se passer si l'arrêt automatique ne fonctionnait pas, s'il était hors service par exemple, et que le conducteur avait un malaise? Où le train irait-il s'arrêter? Là, il y a eu le souci de se dire qu'on allait demander au conducteur d'actionner le cerclo beaucoup plus souvent, c'est-à-dire toutes les $5 \mathrm{~s}$, si bien qu'un conducteur ayant un malaise, avec un arrêt automatique hors service, et franchissant un signal fermé, le train s'immobiliserait au bout de $5 \mathrm{~s}$, puisqu'il faut actionner le système toutes les $5 s^{\prime 12}$. Tout se passe comme si, pour faire face à une panne des automates, il fallait, en contrepartie, obliger le conducteur à bouger tout le temps, comme s'il fallait le mouvement perpétuel de l'agent pour compenser l'immobilité accidentelle des machines.

Ce processus de déréalisation d'un dispositif de sécurité inscrit alors le groupe en charge de le concevoir et de le contrôler dans une forme "d'aliénation culturelle » où "l'impératif de solidarité entre ses membres est placé au-dessus de la prise en considération du réel qui est la raison d'être du groupe. (...) Et avec le temps, un processus s'installe au terme duquel c'est la culture tout entière du groupe qui fait écran à la perception du réel qu'elle est censée représenter" (Sigaut, 1990).

L'imaginaire associé à l'« homme mort » dans le milieu des transports rend assez bien compte de tels processus où l'on voit une multiplicité de théories contradictoires coexister dans des objets techniques qui ne parviennent plus à articuler leur action à leur finalité, leur forme à leur fonction. L'impossibilité pour ces dispositifs de raisonner leur forme trouve probablement son origine, au moins pour partie, dans une perte de capacité d'énonciation d'une de ses justifications initiales, la lutte contre la fraude.

Avec ce refoulement d'une des fonctions du dispositif, celle de la mise en discipline des conducteurs pour lutter contre leur défaillance morale, c'est à

12 Didier Caligny op. cit.

Robin Foot et Ghislaine Doniol-Shaw, « Dérive d'un dispositif » 
une perte de capacité d'articulation des choses aux mots à laquelle on assiste. Sans la ressource des mots pour raisonner les choses, le dispositif convoque d'autres ressources pour justifier de ses évolutions.

Ce peut être un imaginaire infantile comme celle d'une mort crispée ou ce peut être des emprunts faits sur un mode mimétique à d'autres dispositifs. Mais, dorénavant, le réel de la fonction ne peut plus faire retour sur le fonctionnement. C'est la norme qui dit le réel de la situation. Tout ce monde ferroviaire est alors entraîné dans la "dérivée des "solutions sauvages »" (Amalberti et Barriquault, 1999) inventées pour transformer et adapter ce dispositif de sécurité.

Si l'on perçoit désormais la mécanique de cette dérive, il reste à trouver les modalités d'une déprise de cette stratégie « autonome » du dispositif, de son retour dans le « monde ». L'estrangement en fait probablement partie. 


\section{Bibliographie}

Akrich, M., 1987. "Comment décrire les objets techniques?" Techniques et Culture, pp.49-64.

Amalberti, R. et Barriquault, C., 1999. "Fondements et limites du retour d'expérience", Annales des Ponts et Chaussées, pp.67-75.

Callon, M., 1986. "Éléments pour une sociologie de la traduction : la domestication des coquilles St-Jacques et des marins pêcheurs dans la baie de St. Brieuc", L'Année Sociologique, Numéro spécial : La sociologie des Sciences et des Techniques, pp.169-208.

Callon, M. et Latour, B., eds.: 1982, La science telle qu'elle se fait. Une anthologie de la sociologie des sciences de langue anglaise. Paris: Pandore.

Doniol-Shaw, G. et Foot, R., eds.: 2006, Travail de conduite et sécurité des tramways : enjeux pour la conception du poste de conduite. Marnela-Vallée: LATTS/T2C.

Foucault, M., 1966, Les mots et les choses. Archéologie des sciences humaines. Gallimard, Paris.

Foucault, M., 1969, L'archéologie du savoir. Bibliothèque des sciences humaines Gallimard, Paris.

Foucault, M., 1975, Surveiller et punir. Naissance de la prison. Gallimard, Paris.

Foucault, M.: 1994 (1977). "Le jeu de Michel Foucault", in D. Defert et F. Ewald, (eds.), Dits et écrits, Vol. 3, 4 vols., Gallimard, Paris, pp. 298-329.

Foucault, M., 1997, Il faut défendre la société. Gallimard-Seuil, Paris.

Ginzburg, C., 2001, A distance. Neuf essais sur le point de vue en histoire. Trans. P.-A. Fabre. Gallimard, Paris.

Latour, B., 1989, La science en action. Trans. M. Biezunski. texte à l'appui La découverte, paris.

Latour, B., 1992, Aramis, ou l'amour des techniques. La Découverte, Paris.

Latour, B., 1999, Politiques de la nature. Comment faire entrer les sciences en démocratie. La Découverte, Paris. 
Mollard, R., Coblentz, A. et Cabon, P.: 1991. "Détection de l'hypovigilance chez les conducteurs de train", in, Transports et Communication, Paradigme, Caen, pp. 65-71.

Ribeill, G., 1997. "Les conducteurs électriciens : tâtonnements et lenteurs autour de la reconnaissance d'un nouveau métier", Revue d'histoire des chemins de fer, pp.385-398.

Sigaut, F., 1990. "Folie, Réel et technologie", Techniques et culture, 15, pp.167-179.

Sigaut, F.: 1991. "Un couteau ne sert pas à couper mais en coupant. Structure, fonctionnement et fonction dans l'analyse des objets", in, 25 ans d'études technologiques en préhistoire. XI Rencontres internationales d'Archéologie et d'Histoire d'Antibes, APDCA, Juan-les-Pins, pp. 21-34. 